\title{
Review of: "Integration of and visions for community pharmacy in primary health care in Denmark"
}

\author{
Meagen Rosenthal ${ }^{1}$ \\ 1 University of Mississippi
}

Potential competing interests: The author(s) declared that no potential competing interests exist.

This is a very well written manuscript. Thank you for the opportunity to review it. I did note one very long sentence (below), that should be shortened or rephrased. I also noted a number of small grammatical issues that should be addressed.

"This in turn, due to many community pharmacies have increased their sale of care products and free trade products e.g., vitamins, minerals, skin care products and bandages has unfortunately resulted in decreased profits on dispensing and counselling on medicines for all community pharmacies, due to the financial regulation in the gross profit agreement where the amount which the pharmacies can add to the purchase price on each medication they sell have decreased. " 